\title{
New orbital analysis of stars at the Galactic center using speckle holography and orbital priors
}

\author{
Anna Boehle ${ }^{1}$, Rainer Schödel ${ }^{2}$, Leo Meyer ${ }^{1}$ and Andrea M. Ghez ${ }^{1}$ \\ ${ }^{1}$ Department of Physics and Astronomy, UCLA, Los Angeles, CA 90095-1547, USA \\ email: aboehle@astro.ucla.edu \\ ${ }^{2}$ Istituto de Astrofisica de Andalucia (CSIC), Glorieta de la Astronomia S/N, E-18008 \\ Granada, Spain
}

\begin{abstract}
We present initial results of a study that has more than doubled the time baseline for astrometric measurements of faint stars orbiting the supermassive black hole (SMBH) at the Galactic center. The advent of adaptive optics has enabled stars as faint as $K=19$ mag to be tracked at 50 mas resolution for the last decade. While similar resolution images exist from the prior decade, they were obtained from speckle imaging data analyzed with the technique of shift-and-add, which limited detections to stars brighter than $K=16$ mag. By improving the speckle data analysis technique with speckle holography and using prior orbital knowledge, we are now able to track stars as faint as $\sim 18$ mag at 50 mas resolution through the early Keck speckle data sets (1995-2005). This methodology has already led to the detection of two shortperiod stars never previously seen in speckle images, such that our data now spans their full orbits. We can now better constrain the orbital parameters of all stars in the intriguing "S-star cluster," which will ultimately give us insight into the origin of these stars and be used to probe the curvature of space-time in the unexplored regime near a SMBH.
\end{abstract}

Keywords. Galaxy: center, Galaxy: kinematics and dynamics, infrared: stars

\section{Introduction}

Since 1995, our group has been monitoring the stars orbiting the supermassive black hole (Sgr $\left.\mathrm{A}^{*}\right)$ at the center of the Milky Way. By modeling the motion of the bright $(K=$ 14 mag), short-period ( $\mathrm{P}=16$ years) star S0-2 as a Keplerian orbit, the mass of Sgr A* and the distance between us and the Galactic center $(\mathrm{GC})\left(\mathrm{R}_{o}\right)$ can be measured. As of now observations have entirely covered the orbit of S0-2, making it the most powerful probe of the central gravitational potential in which the stars in the central region of our galaxy move (see Ghez et al. 2008 and Gillessen et al. 2009).

The addition of more short-period stars with observations covering a high fraction of their orbit can be used to further constrain the mass of Sgr A* and $\mathrm{R}_{o}$. We present here a new methodology to detect stars in our first 10 years of speckle imaging data, doubling the observational time baseline of faint stars and the successful test of this methodology on the short-period star $\mathrm{S} 0-38$.

\section{New methodology for the analysis of speckle imaging data and results on the short-period star, S0-38}

We use two sets of imaging data to determine the positions of stars over time: speckle imaging data (taken from 1995 - 2005) and adaptive optics (AO) data (taken from 2005 - 2013). We have recently re-reduced our speckle imaging data using a newly developed 

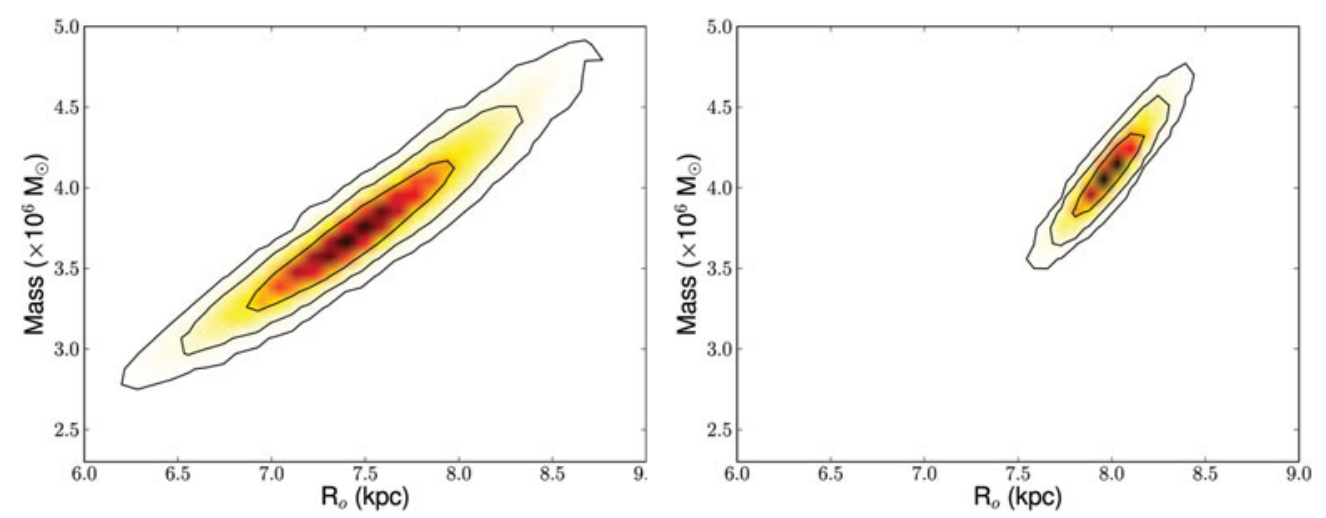

Figure 1. 2D joint probability distribution with 1-, 2-, and 3-4 $\sigma$ contours of mass of Sgr A* and $\mathrm{R}_{o}$ as derived by orbital fit of S0-2 alone (left) and by a simultaneous fit of S0-2 and S0-38, with the new speckle detections of S0-38 included (right). The precision of our measurements of mass and $\mathrm{R}_{o}$ has increased by factors of 2 and 3 respectively. (Note that these distributions do not include uncertainties in our absolute reference frame.) [A COLOR VERSION IS AVAILABLE ONLINE.]

technique called speckle holography, which involves the deconvolution of the individual short-exposure images with the instantaneous point spread function (see Schödel et al. 2013). This technique yields higher quality and deeper maps than the shift-and-add technique we originally used. It has improved the median $K$-band limiting magnitude of our speckle images from $K=15.9$ to $17.0 \mathrm{mag}$.

In order to search for faint stars in the speckle holography data sets, we have developed a new method that uses the knowledge we have about a star's orbit from our 9-year AO data set. Since our AO data are so much deeper than the speckle holography data, any star we could possibly detected in the speckle holography epochs is easily detected and tracked over the AO data set. This methodology takes advantage of this fact by using the probability of the position of a given star in each holography epoch to inform the search for the star in that image.

This use of orbital prior information to search for faint stars in the speckle data along with the speckle holography reduction method has allowed us to find stars never before detected in the first 10 years of our imaging data. This new analysis more than doubles our time baseline for these faint stars. We have successfully applied the new methodology on the short-period ( $\mathrm{P}=19$ years) star $\mathrm{S} 0-38$. With a $K$-band magnitude of $17.0, \mathrm{~S} 0-38$ was not detected in the original analysis of speckle data. Using our new methodology we have now detected S0-38 in 12 new epochs. The addition of these new detections to the $\mathrm{AO}$ detections has extended the time baseline of our data on $\mathrm{S} 0-38$ such that they now cover more than $90 \%$ the stars full orbit. S0-38 now joins S0-2 and the newly-discovered S0-102 (Meyer et al. 2012) as one of only three stars with orbital phase coverage more than $50 \%$. Thanks to its high orbital phase coverage, S0-38 can now be used along with $\mathrm{S} 0-2$ as an additional constraint on the mass of Sgr $\mathrm{A}^{*}$ and $\mathrm{R}_{o}$. Figure 1 compares the joint probability distribution of mass and $\mathrm{R}_{o}$ for the fit of the $\mathrm{S} 0-2$ data alone and for the simultaneous fit of the S0-2 and S0-38 data together. With the addition of S0-38, the precision of our mass and $\mathrm{R}_{o}$ measurements have increased by a factor 2 and 3 respectively. (Note that the errors for mass and $\mathrm{R}_{o}$ quoted here do not include uncertainties in our absolute reference frame.) 


\section{References}

Ghez, A. M., et al. 2008, ApJ 689, 1044

Gillessen, S., et al. 2009, ApJ 692, 1075

Meyer, L., Ghez, A. M., Schödel, R., Boehle, A., et al. 2012, Science 338, 84

Schödel, R., Yelda, S., Ghez, A. M., et al. 2013, MNRAS 429, 1367 